Ilchmann, Achim; Townley, Stuart; Thuto, Mosalagae :

Adaptive sampled-data tracking for input-constrained exothermic chemical reaction models

Zuerst erschienen in:

Systems \& Control Letters, 54 (2005), Nr. 12, S. 1149-1161 DOI: 10.1016/i.sysconle.2005.04.004 


\title{
Adaptive sampled-data tracking for input-constrained exothermic chemical reaction models
}

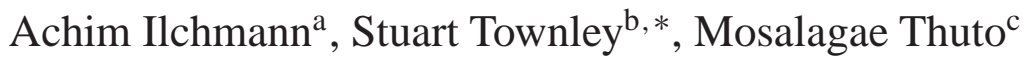 \\ a Institut für Mathematik, Technische Universität Ilmenau, Weimarer Straße 25, 98693 Ilmenau, DE \\ ${ }^{\mathrm{b}}$ School of Engineering, Computer Science and Mathematics, University of Exeter, North Park Road, Exeter EX4 4QE, UK \\ ${ }^{\mathrm{c}}$ Department of Mathematics, University of Botswana, Private Bag 0022, Botswana
}

Received 8 September 2004; received in revised form 7 April 2005; accepted 14 April 2005

\begin{abstract}
We consider digital input-constrained adaptive and non-adaptive output feedback control for a class of nonlinear systems which arise as models for controlled exothermic chemical reactors. Our objective is set-point control of the temperature of the reaction, with pre-specified asymptotic tracking accuracy set by the designer. Our approach is based on $\lambda$-tracking controllers, but in a context of piecewise constant sampled-data output feedbacks and possibly adapted sampling periods. The approach does not require any knowledge of the systems parameters, does not invoke an internal model, is simple in its design, copes with noise corrupted output measurements, and requires only a feasibility assumption in terms of the reference temperature and the input constraints.
\end{abstract}

(C) 2005 Elsevier B.V. All rights reserved.

Keywords: Adaptive control; Exothermic chemical reactors; Input saturation; Sampling; Tracking; Global stabilisation

\section{Introduction}

We consider adaptive sampled-data control of input-constrained continuous-stirred, exothermic chemical reactor models. The main issues in this context include: typically reactor models are highly nonlinear; the rate of conversion of reactant into product should be economically profitable; quite often the operating point is close to an open-loop unstable hyperbolic equilibrium; there is model uncertainty, inputs are saturated; and measured data can be corrupted by noise.

The area of high-gain adaptive control without identification was initiated by the seminal papers $[12,2,10]$. Practical tracking in the sense of $\lambda$-tracking has been introduced by [11,4]. Sampled data control in conjunction

\footnotetext{
* Corresponding author. Tel.: +44 1392263975 ; fax: +44 1392263997.

E-mail addresses: ilchmann@mathematik.tu-ilmenau.de (A. Ilchmann), townley@maths.ex.ac.uk (S. Townley), thutomv@mopipi.ub.bw (M. Thuto).
} 
with $\lambda$-tracking has been investigated in [6] but only for linear systems and also without input saturation. A brief review of the relevant literature on exothermic chemical reactors and their regulation can be found in [1,9]. In [5], we developed simple adaptive controllers for a class of nonlinear reactor models of a type similar to those considered in [9]. We showed that the controllers could meet the control objectives mentioned above. However, several open problems remained. Amongst these, we have focused on two: (i) Is it possible to prespecify the closedloop performance? (ii) Does digital (i.e. sampled-data) implementation cause any difficulties? The former has been addressed in [7] in a context of funnel control. The latter is investigated in the current note.

The need for adapting the sampling period, instead of simply setting the sampling rate as fast as possible, can be justified in various ways: A striking example is in insulin injection, where fast sampling/injection would be disadvantageous both for the patient and the doctor; in process control and electrical engineering, taking precise measurements can be expensive, whilst recording too many measurements may be inpractical. Also, industrial processors have limited capacity, and so with less frequent sampling, the processor can be used for calculations other than taking samples; high frequency usage of actuators, due to very fast sampling rates, can be expensive or may lead to frequent actuator failure.

In this paper, we focus on an adaptive scheme with both feedback gain and sampling rate adaptation. As a byproduct we obtain non-adaptive feedback strategies which accordingly require sufficiently large/small and constant gains/sampling periods, respectively.

\subsection{System class}

As in $[9,5]$, we consider coupled reactant-product-temperature models of the form

$$
\begin{gathered}
\dot{x}_{1}(t)=C^{1} r(x(t), T(t))+d\left[v(t)-x_{1}(t)\right], \quad x_{1}(0)=x_{1}^{0} \in \mathbb{R}_{\geqslant 0}^{n-m}, \\
\dot{x}_{2}(t)=C^{2} r(x(t), T(t))+d\left[x_{2}^{\text {in }}-x_{2}(t)\right], \quad x_{2}(0)=x_{2}^{0} \in \mathbb{R}_{\geqslant 0}^{m}, \\
\dot{T}(t)=b^{\mathrm{T}} r(x(t), T(t))-q T(t)+u(t), \quad T(0)=T^{0} \in \mathbb{R}_{>0} .
\end{gathered}
$$

In (1) the variables and constants represent, for $n, m \in \mathbb{N}$ with $0<m<n$, the following:

$$
\begin{aligned}
& x(t)=\left(\begin{array}{l}
x_{1} \\
x_{2}
\end{array}\right) \in \mathbb{R}_{\geqslant 0}^{n} \\
& \text { concentration of chemical reactants and products at time } t \geqslant 0 \\
& x_{1}(t) \in \mathbb{R}_{\geqslant 0}^{n-m} \\
& x_{2}(t) \in \mathbb{R}_{\geqslant 0}^{m} \\
& T(t) \in \mathbb{R}_{>0} \\
& x^{\text {in }}=\left(\begin{array}{c}
x_{1}^{\text {in }} \\
x_{2}^{\text {in }}
\end{array}\right) \in \mathbb{R}_{\geqslant 0}^{(n-m)+m} \\
& \text { concentrations of the } n-m \text { chemical reactants at time } t \geqslant 0 \\
& \text { concentrations of the } m \text { chemical products at time } t \geqslant 0 \\
& \text { temperature of the reactor at time } t \geqslant 0 \\
& \text { constant feed concentrations } \\
& v(t) \in\left[0, x_{1}^{\mathrm{in}}\right] \\
& u(t) \in \mathbb{R} \geqslant 0 \\
& C=\left(\begin{array}{l}
C^{1} \\
C^{2}
\end{array}\right) \in \mathbb{R}^{n \times m} \\
& C^{1} \in \mathbb{R}_{\leqslant 0}^{(n-m) \times m} \\
& b \in \mathbb{R}_{\geqslant 0}^{m} \\
& d>0 \\
& \text { control of the cooling at time } t \geqslant 0 \\
& \text { control of the temperature at time } t \geqslant 0 \\
& \text { stoichiometric matrix } \\
& \text { stoichiometric matrix of the reactants, accordingly all entries of } C^{1} \text { are non-positive } \\
& \text { coefficients of the exothermicity } \\
& \text { dilution rate } \\
& q>0 \\
& \text { heat transfer rate between heat exchanger and reactor } \\
& r(\cdot, \cdot): \mathbb{R}_{\geqslant 0}^{n} \times \mathbb{R}_{>0} \rightarrow \mathbb{R}_{\geqslant 0}^{m} \text { a locally Lipschitz continuous function modelling the reaction kinetics }
\end{aligned}
$$


System (1) and the following assumptions (A1)-(A4) capture the essential features of exothermic chemical reactor models:

(A1) $\mathbb{R}_{\geqslant 0}^{n} \times \mathbb{R}_{>0}$ is positively invariant under (1) for any bounded, non-negative, piecewise constant functions $u(\cdot): \mathbb{R} \geqslant 0 \rightarrow \mathbb{R} \geqslant 0$ and $v(\cdot): \mathbb{R} \rightarrow\left[0, x_{1}^{\text {in }}\right]$.

(A2) There exists $\gamma \in \mathbb{R}_{>0}^{n}$ such that $\gamma^{\mathrm{T}} c_{i} \leqslant 0$ for all columns $c_{1}, \ldots, c_{m}$ of the stoichiometric matrix $C$.

(A3) For $T^{*}>0$ there exist $0<\underline{T}<T^{*}<\bar{T}, \rho>0,0<\underline{u}<\bar{u}$, such that

$$
0<\underline{u}+\rho<q T-b^{\mathrm{T}} r(x, T)<\bar{u}-\rho \quad \forall(x, T) \in \Omega\left(\gamma, x^{\mathrm{in}}\right) \times[\underline{T}, \bar{T}] .
$$

where

$$
\mathbf{\Omega}\left(\gamma, \mathbf{x}^{\text {in }}\right):=\left\{x \in \mathbb{R}_{>0}^{n} \mid \gamma^{\mathrm{T}} x<\gamma^{\mathrm{T}} x^{\mathrm{in}}\right\} .
$$

$$
\|r(x, T)\| \leqslant \hat{r}\left(x_{1}\right) T \quad \forall(x, T)=\left(\left(x_{1}^{\mathrm{T}}, x_{2}^{\mathrm{T}}\right)^{\mathrm{T}}, T\right) \in \Omega\left(\gamma, x^{\mathrm{in}}\right) \times \mathbb{R}_{>0}
$$

for some continuous function $\hat{r}: \mathbb{R}_{\geqslant 0}^{n-m} \rightarrow \mathbb{R}_{\geqslant 0}$ with $\lim _{x_{1} \rightarrow 0} \hat{r}\left(x_{1}\right)=0$.

Assumption (A1) is natural for exothermic reactions. Indeed, concentrations and temperature should not become zero once they are positive. In fact, since $r(\cdot, \cdot)$ is non-negative, if $u(\cdot)$ is non-negative, then it is clear that $T(t)>0$ whenever $T^{0}>0$. It is easy to show that the remainder of (A1) holds automatically when $n=2$, i.e., in the case of a single reaction. For multiple reactions, there are various conditions (see, e.g., [8, Proposition 6]) in terms of specific rates which imply that (A1) holds.

Assumption (A1) has been formulated for the closed positive orthant $\mathbb{R}_{\geqslant 0}^{n}$ of the concentrations and the open half line for the temperature. The latter is natural since the reactor should not operate with zero or negative temperature; the former could also be assumed for the open positive orthant $\mathbb{R}_{>0}^{n}$; the analysis goes through without any changes.

Assumption (A2) holds if (1) satisfies the law of conservation of mass, which means that there exists $\gamma \in \mathbb{R}_{>0}^{n}$ with $\gamma^{\mathrm{T}} C=0$. This can be found implicitly in Gavalas [3], and it is also assumed in Viel et al. [14]. If $C$ does not represent exactly the stoichiometric relationships between all species, then conservation of mass need not be satisfied. Nevertheless, the reaction model might still be relevant provided that all essential reactions are obeyed. This approach was adopted in Bastin and Dochain [1] and also in [8]. A concept of "noncyclic processes" is developed in the latter article to ensure dissipativity of mass, and hence that (A2) is satisfied.

Assumption (A3) guarantees "feasibility" by relating the temperature setpoint $T^{*}$ and the positive input saturations $\underline{u}$ and $\bar{u}$ to weak system data. This assumption could be formulated less technically as

$\left(\mathrm{A}^{\prime}\right)$ For $T^{*}>0$ there exist $0<\underline{u}<\bar{u}$, such that $\underline{u}<q T^{*}-b^{\mathrm{T}} r\left(x, T^{*}\right)<\bar{u} \quad \forall x \in \Omega\left(\gamma, x^{\mathrm{in}}\right)$,

which implies (A3) for suitable $\underline{T}, \bar{T}, \rho$. However, the more explicit assumption (A3) is easier to use, and the introduction of $\rho$ makes the exposition in the proofs clearer. Note that (A3') coincides with (H3) in [14].

Finally, assumption (A4) encompasses multi-reaction kinetics considered in [14] and guarantees in particular that the reaction kinetics are zero if the temperature is zero.

\subsection{Control objective}

The control objective is to regulate the temperature $T(t)$ towards a pre-specified neighbourhood of size $\lambda>0$ of a given reference temperature $T^{*}$, whilst maintaining boundedness of all variables. In achieving this objective, we are restricted to using only temperature measurements which may be corrupted by bounded noise $n(\cdot): \mathbb{R} \geqslant 0 \rightarrow \mathbb{R}$, so that the measured error becomes

$$
e(t)=T^{*}-T(t)+n(t) .
$$


We stress that the noise need not be smooth but its norm has to be sufficiently small in terms of $\lambda$, i.e. the accuracy of the tracking error, and in terms of $\bar{T}-T^{*}$. This will be specified in (10) and it is needed basically to ensure that a feedback based on disturbed outputs can recognize the difference between $T^{*}$ and $\bar{T}$.

Not only do we want to cope with noise corrupted measurements but additionally we want the controller to be implemented digitally so that we have access to these corrupted measurements only at sampled time instants. Specifically, we use zero-order sampling

$$
T_{i}:=T\left(t_{i}\right)
$$

and zero-order hold

$$
u(t):=u\left(t_{i}\right) \text { for all } t \in\left[t_{i}, t_{i+1}\right),
$$

where the sampled time is $t_{i}, i \in \mathbb{N}$, and $t_{0}=0$.

\subsection{Adaptive sampled-data controller}

We introduce a rather simple control strategy. It consists merely of a monotonically non-decreasing gain adaptation (5), depending on the distance of the error to the pre-specified $\lambda$-strip; a decreasing sampling adaptation (4), tuned by the gain which increases as long as the measured error $e_{i}$ is outside the $\lambda$-strip; and a zero-order hold feedback (6) which is piecewise constant on the sampling intervals and obeys input saturations. The flexibility of selecting suitable functions $f$ and $g_{\lambda}$ could be useful in applications.

For initial values $t_{0}=0, \beta_{0}>0$, and input offset $u^{*} \in(\underline{u}, \bar{u})$, we define the adaptive sample and hold controller, for all $i \in \mathbb{N}_{0}$, as follows:

Sampling:

$$
e_{i}:=e\left(t_{i}\right) .
$$

Sampling rate adaptation:

$$
h_{i}=f\left(\beta_{i}\right), \quad t_{i+1}=t_{i}+h_{i},
$$

where $f(\cdot): \mathbb{R}_{\geqslant 0} \rightarrow \mathbb{R}_{>0}$ is a non-increasing function with $\lim _{\beta \rightarrow \infty} f(\beta)=0$.

Gain adaptation:

$$
\beta_{i+1}=\beta_{i}+h_{i} g_{\lambda}\left(e_{i}\right)
$$

where, for $\lambda>0$, the continuous function $g_{\lambda}: \mathbb{R} \rightarrow \mathbb{R} \geqslant 0$ satisfies

$$
\begin{aligned}
& \forall e \in \mathbb{R}:\left[g_{\lambda}(e)=0 \Longleftrightarrow|e| \leqslant \lambda\right] \\
& \liminf _{e \rightarrow \infty} g_{\lambda}(e)>0
\end{aligned}
$$

Zero-order hold feedback:

$$
\begin{aligned}
& u(t):=u_{i}=\operatorname{sat}_{[\underline{u}, \bar{u}]}\left(\beta_{i} e_{i}+u^{*}\right), \\
& v(t):=v_{i}=\left\{\begin{array}{ll}
0 & \text { if } \beta_{i} e_{i} \leqslant \underline{u}-u^{*} \\
x_{1}^{\text {in }} & \text { else }
\end{array}\right\} t \in\left[t_{i}, t_{i+1}\right) .
\end{aligned}
$$


An example of the control strategy (3)-(6) is, for $p \geqslant 1, \alpha, \beta_{0}>0$, and $t_{0}=0$,

$$
\begin{aligned}
& h_{i}=\left(1+\beta_{i}\right)^{-\alpha}, \quad t_{i+1}=t_{i}+h_{i}, \quad e_{i}=T^{*}-T\left(t_{i}\right)+n\left(t_{i}\right), \\
& \beta_{i+1}=\beta_{i}+h_{i} \max \left\{\left|e_{i}\right|-\lambda, 0\right\}^{p}, \\
& u(t)=\operatorname{sat}_{[\underline{u}, \bar{u}]}\left(\beta_{i} e_{i}+u^{*}\right), \quad t \in\left[t_{i}, t_{i+1}\right), \\
& v(t)=\left\{\begin{array}{ll}
0 & \text { if } \beta_{i} e_{i} \leqslant \underline{u}-u^{*} \\
x_{1}^{\text {in }} & \text { else }
\end{array} \quad t \in\left[t_{i}, t_{i+1}\right) .\right.
\end{aligned}
$$

\subsection{Non-adaptive sampled-data controller}

For initial time $t_{0}=0$, gain $\beta>0$, sampling period $h>0$, and input offset $u^{*} \in(\underline{u}, \bar{u})$, we define, for all $i \in \mathbb{N}_{0}$, the sampling by

$$
e_{i}:=e\left(t_{i}\right), \quad t_{i}:=h i,
$$

and the non-adaptive sample and hold controller by

$$
\begin{aligned}
& u(t):=u_{i}=\operatorname{sat}_{[\underline{u}, \bar{u}]}\left(\beta e_{i}+u^{*}\right), \\
& v(t):=v_{i}=\left\{\begin{array}{ll}
0 & \text { if } \beta e_{i} \leqslant \underline{u}-u^{*} \\
x_{1}^{\text {in }} & \text { else }
\end{array}\right\} \quad t \in\left[t_{i}, t_{i+1}\right) .
\end{aligned}
$$

Note that in both control strategies (6) and (9) we have two control actions: a saturating control of the temperature and an on/off control of reactant feed.

\section{Main results}

We are now in a position to state the main result which, loosely speaking, shows that, under the standard chemical reaction model assumptions (A1)-(A4), temperature setpoint tracking can be achieved with prespecified accuracy $\lambda>0$ by the simple sampled-data control strategy (3)-(6): the sampled temperature $T_{i}$ tends to the $\left[\lambda+\|n\|_{\infty}\right]$-strip $\left[T^{*}-\left[\lambda+\|n\|_{\infty}\right], T^{*}+\left[\lambda+\|n\|_{\infty}\right]\right]$ as $i \rightarrow \infty$; moreover, all signals are bounded, and convergence of the gain and sampling period is ensured. We stress that the controller tolerates (not necessarily smooth) noise corrupting the output measurement as long as the noise is sufficiently small.

Global/local and adaptive/non-adaptive versions of this result are presented. All proofs are deferred to Section 4.

Theorem 1 (Global adaptive result). Consider (1) satisfying (A1)-(A4), let $\lambda>0$, and $n(\cdot): \mathbb{R} \geqslant 0 \rightarrow \mathbb{R}$ be a function satisfying

$$
2\|n\|_{\infty}<\min \left\{\bar{T}-T^{*}, \lambda\right\} .
$$

Then the application of the sampled-data adaptive controller (3)-(6) to (1) yields, for any initial data $\left(x^{0}, T^{0}, \beta_{0}\right) \in$ $\Omega\left(\gamma, x^{\text {in }}\right) \times \mathbb{R}_{>0} \times \mathbb{R}_{>0}$, an initial value problem which has a unique solution

$$
(x(\cdot), T(\cdot)): \mathbb{R}_{\geqslant 0} \longrightarrow \Omega\left(\gamma, x^{\text {in }}\right) \times \mathbb{R}_{>0}
$$

and this solution satisfies

(i) $T(\cdot)$ is bounded;

(ii) $\beta_{i} \uparrow \beta_{\infty} \in \mathbb{R}_{>0}$ as $i \rightarrow \infty$; 
(iii) $h_{i} \downarrow h_{\infty} \in \mathbb{R}_{>0}$ as $i \rightarrow \infty$;

(iv) $\lim _{i \rightarrow \infty} \operatorname{dist}\left(\left|T^{*}-T_{i}\right|,\left[0, \lambda+\|n\|_{\infty}\right]\right)=0$.

Note that if $n(\cdot) \equiv 0$, then (10) is redundant. In this sense, our controller is robust with respect to sufficiently small output measurement noise.

The following result is a non-adaptive version of Theorem 1 which, whilst preserving the simplicity of the control strategy, requires the sampling period $h$ to be sufficiently small and the gain $\beta$ to be sufficiently large.

Theorem 2 (Global non-adaptive result). Consider (1) satisfying (A1)-(A4), let $\lambda>0$, and $n(\cdot): \mathbb{R} \geqslant 0 \rightarrow \mathbb{R}$ be a function satisfying (10). Let $g_{\lambda}: \mathbb{R} \rightarrow \mathbb{R} \geqslant 0$ be continuous function satisfying (5a) and (5b). For any (1) with initial data $\left(x^{0}, T^{0}\right) \in \Omega\left(\gamma, x^{\text {in }}\right) \times \mathbb{R}_{>0}$, there exist sufficiently small $h^{*}>0$ and sufficiently large $\beta^{*}>0$ such that the application of the sampled-data controller (8), (9), for any $h \in\left(0, h^{*}\right)$ and $\beta>\beta^{*}$, yields an initial value problem which has a unique solution

$$
(x(\cdot), T(\cdot)): \mathbb{R}_{\geqslant 0} \longrightarrow \Omega\left(\gamma, x^{\text {in }}\right) \times \mathbb{R}_{>0}
$$

and this solution satisfies that $T(\cdot)$ is bounded and

$$
\lim _{i \rightarrow \infty} \operatorname{dist}\left(\left|T^{*}-T_{i}\right|,\left[0, \lambda+\|n\|_{\infty}\right]\right)=0 .
$$

Consider again the control strategies (6) and (9). If the upper feasibility bound $\bar{T}$ is known, and additionally we have that the initial temperature is below this bound, then we only need to use saturated control $u(\cdot)$ of temperature. In the absence of additional cooling action we then have a model

$$
\begin{aligned}
& \dot{x}(t)=C r(x(t), T(t))+d\left[x^{\text {in }}-x(t)\right], \quad x(0)=x^{0} \in \mathbb{R}_{\geqslant 0}^{n}, \\
& \dot{T}(t)=b^{\mathrm{T}} r(x(t), T(t))-q T(t)+u(t), \quad T(0)=T^{0} \in \mathbb{R}_{>0} .
\end{aligned}
$$

Proposition 3 (Local result, adaptive and non-adaptive). Consider (11) satisfying (A1)-(A3), let $\lambda>0$, and $n(\cdot)$ : $\mathbb{R} \geqslant 0 \rightarrow \mathbb{R}$ be a function satisfying (10).

(i) Then the application of the sampled-data adaptive controller (3)-(6) to (11) yields, for any initial data $\left(x_{0}, T_{0}\right) \in$ $\Omega\left(\gamma, x^{\mathrm{in}}\right) \times(0, \bar{T}]$, and $\beta_{0}$ sufficiently large, an initial value problem which has a unique solution

$$
(x(\cdot), T(\cdot)): \mathbb{R} \geqslant 0 \longrightarrow \Omega\left(\gamma, x^{\text {in }}\right) \times(0, \bar{T})
$$

and this solution satisfies the Statements (i)-(iv) of Theorem 1.

(ii) Let $g_{\lambda}: \mathbb{R} \rightarrow \mathbb{R} \geqslant 0$ be continuous function satisfying (5a) and (5b). For any (1) with initial data $\left(x^{0}, T^{0}\right) \in$ $\Omega\left(\gamma, x^{\text {in }}\right) \times \mathbb{R}_{>0}$, there exists sufficiently small $h^{*}>0$ and sufficiently large $\beta^{*}>0$ such that the application of the sampled-data controller (8), (9), for any $h \in\left(0, h^{*}\right)$ and $\beta>\beta^{*}$, yields an initial value problem which has a unique solution

$$
(x(\cdot), T(\cdot)): \mathbb{R} \geqslant 0 \longrightarrow \Omega\left(\gamma, x^{\mathrm{in}}\right) \times(0, \bar{T})
$$

and this solution satisfies

$$
\lim _{i \rightarrow \infty} \operatorname{dist}\left(\left|T^{*}-T_{i}\right|,\left[0, \lambda+\|n\|_{\infty}\right]\right)=0 .
$$

Remark 4 (Mixed adaptive/non-adaptive gain/sampling). Analogous to Theorem 2 and Proposition 3 we could also show that a mixed adaptive sampling and non-adaptive gain, or non-adaptive sampling and adaptive gain achieves the control objectives in case of the global or local set-up, provided the constants $h^{*}$ and $\beta^{*}$ are sufficiently small and large, respectively. We leave this out for brevity. 
Remark 5 (Intersampling behaviour). For the intersampling behaviour of all the control strategies applied in Theorems 1 and 2, Proposition 3, or the ones mentioned in Remark 4 the following estimates hold: For any

$$
M>\tilde{M}:=\max \left\{\|b\| \hat{r}\left(x_{1}\right)-q \mid\left(x_{1}, x_{2}\right) \in \Omega\left(\gamma, x^{\text {in }}\right)\right\}
$$

we have

$$
\mathrm{e}^{-q h_{i}} T_{i} \leqslant T(t) \leqslant \mathrm{e}^{M h_{i}} T_{i}+\frac{\mathrm{e}^{M h_{i}}-1}{M} \bar{u} \quad \forall i \in \mathbb{N} \forall t \in\left[t_{i}, t_{i+1}\right) .
$$

Note that $\tilde{M} \geqslant 0$ is well defined since $x_{1}$ lies in a bounded set and $\hat{r}$ is continuous. Then Assumption (A4) yields

$$
-q T(t)+\underline{u} \leqslant \dot{T}(t) \leqslant\|b\| \hat{r}\left(x_{1}\right) T(t)-q T(t)+\bar{u} \leqslant M T(t)+\bar{u},
$$

and integration gives, for all $i \in \mathbb{N}$ and all $t \in\left[t_{i}, t_{i+1}\right)$,

$$
\mathrm{e}^{-q\left(t-t_{i}\right)} T\left(t_{i}\right)+\int_{t_{i}}^{t} \mathrm{e}^{-q(t-s)} \underline{u} \mathrm{~d} s \leqslant T(t) \leqslant \mathrm{e}^{M\left(t-t_{i}\right)} T\left(t_{i}\right)+\int_{t_{i}}^{t} \mathrm{e}^{M(t-s)} \bar{u} \mathrm{~d} s,
$$

whence (13). However, the estimate (13) of the intersampling behaviour is conservative and, more importantly, (12) requires knowledge or bounds on the system data which is excluded in our setup.

\section{Example and simulations}

In this section, we consider the special case of a single reaction so that (1) is of the form

$$
\begin{aligned}
\dot{x}_{1}(t) & =-k(T(t)) x_{1}(t)+d\left[v(t)-x_{1}(t)\right], \quad x_{1}(0)=x_{1}^{0} \in \mathbb{R} \geqslant 0, \\
\dot{x}_{2}(t) & =k(T(t)) x_{1}(t)+d\left[x_{2}^{\text {in }}-x_{2}(t)\right], \quad x_{2}(0)=x_{2}^{0} \in \mathbb{R} \geqslant 0, \\
\dot{T}(t) & =b k(T(t)) x_{1}(t)-q T(t)+u(t), \quad T(0)=T^{0} \in \mathbb{R}_{>0} .
\end{aligned}
$$

Here $b>0$ denotes the exothermicity of a reaction $A \longrightarrow B, x^{\text {in }}=\left(x_{1}^{\text {in }}, 0\right)^{\mathrm{T}}$, where $x_{1}^{\text {in }}$ is the constant feed rate of reactant $A$, and the reaction kinetics are given by a locally Lipschitz function $k(\cdot): \mathbb{R} \geqslant 0 \rightarrow \mathbb{R} \geqslant 0$ with $k(0)=0$. A typical example of $k(\cdot)$ is the Arrhenius law $T \mapsto k_{0} \exp \{-E / R T\}$ (extended to zero by continuity), where $k_{0} \in \mathbb{R}_{>0}$ is a constant, $E$ is the activation energy, and $R$ is the Joule constant. The parameters of the function $k(\cdot)$ and the positive constants $d, q$, and $b$ are typically unknown.

This prototypical example is also studied in [14] and in [5]. Here we choose the same system parameters—consistent with a laboratory-scale reaction vessel of approximately 1001 - so that simulations can be compared.

$$
\begin{aligned}
& T \mapsto k(T)=k_{0} \mathrm{e}^{-k_{1} \mathrm{~T}}, \quad k_{0}=\mathrm{e}^{25}, \quad k_{1}=8700 \mathrm{~K}, \\
& d=1.1, \quad q=1.25 \mathrm{~min}^{-1}, \quad x_{1}^{\text {in }}=x_{2}^{\text {in }}=1 \mathrm{~mol} / 1, \quad b=209.2 \mathrm{Kl} / \mathrm{mol} .
\end{aligned}
$$

The controller (7) with parameters

$$
\begin{array}{ll}
\underline{u}=295, & \bar{u}=505, \quad u^{*}=330, \quad T^{*}=337.1 \mathrm{~K}, \\
\beta^{0}=12, & p=2, \quad \alpha=1, \quad \lambda=2.85,
\end{array}
$$

is applied to (14) with initial conditions

$$
x_{1}^{0}=0.02, \quad x_{2}^{0}=1.07, \quad T^{0} \in\{270,320,390\},
$$

and disturbance signal

$$
n(t)=q_{1}(t) / 15,
$$



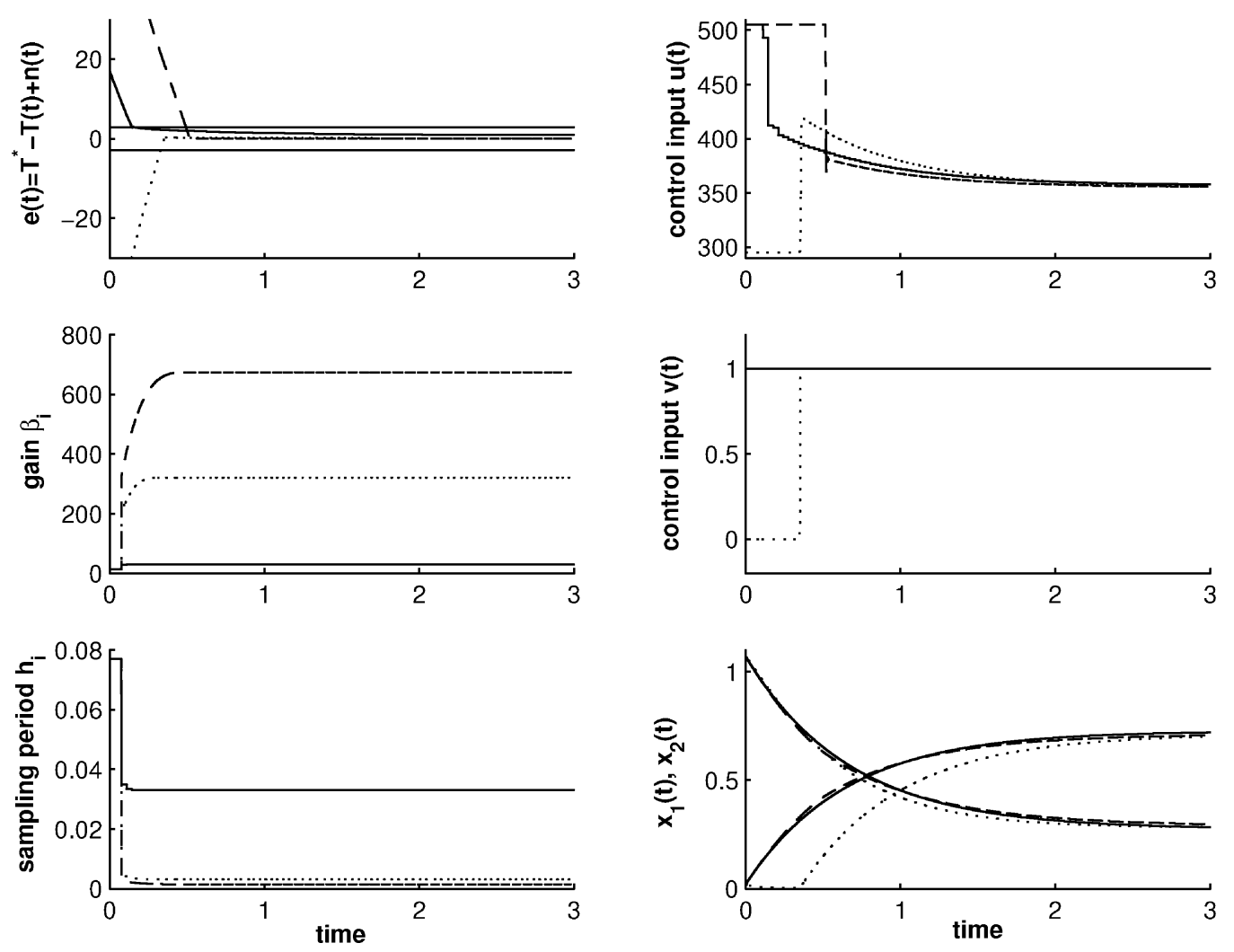

Fig. 1. Closed-loop setpoint control of the adaptive $\lambda$-tracker (7) with control parameters (16) applied to the single reaction (14) with parameters (15) and initial temperature $T^{0}=270 \mathrm{~K}$ (dashed), $T^{0}=320 \mathrm{~K}$ (solid), $T^{0}=390 \mathrm{~K}$ (dotted).

where $q_{1}(\cdot)$ is the first component of the Lorenz equation

$$
\frac{\mathrm{d}}{\mathrm{d} t}\left(q_{1}, q_{2}, q_{3}\right)=\left(10\left[q_{2}-q_{1}\right], 28 q_{1}-q_{2}-q_{1} q_{3}, q_{1} q_{2}-\frac{8}{3} q_{3}\right), \quad\left(q_{1}, q_{2}, q_{3}\right)(0)=(1,0,3) .
$$

This Lorenz equation is known Sparrow [13] to exhibit chaotic but bounded behaviour. In this case $|n(t)| \leqslant 1.25$ for all $t \geqslant 0$.

The objective is to regulate the temperature to a neighbourhood of $T^{*}=337.1 \mathrm{~K}$. It is easy to see that for

$$
\gamma=(1,1)^{\mathrm{T}}, \quad \underline{T}=240, \quad \bar{T}=339.65 \mathrm{~K}, \quad \rho=5,
$$

the inequality in (10) and the feasibility assumption (A3) are satisfied for some $\rho>0$.

All the above parameters are the same as for the simulations in [5], and the sampled data controller is the Euler approximation of the continuous time controller. Figs. 1 and 2 depict sampled-data simulations corresponding to their continuous time counterparts, which can be seen in Figs. 2 and 3 from [5].

In [5] we have shown by simulations that for initial temperature $T^{0}=390 \notin[0, \bar{T}]$, the local controller cannot cope: there is a thermal runaway and the temperature is attracted to a stable but undesirably high temperature. As a result, the reaction becomes overheated, the reactant burns out, and there is a rapid growth of the product. Furthermore, the control input saturates at its lower limit throughout the simulation and the gain increases unboundedly. The same would be true for the sampled-data result as in Proposition 3. We have omitted this simulation for brevity. 

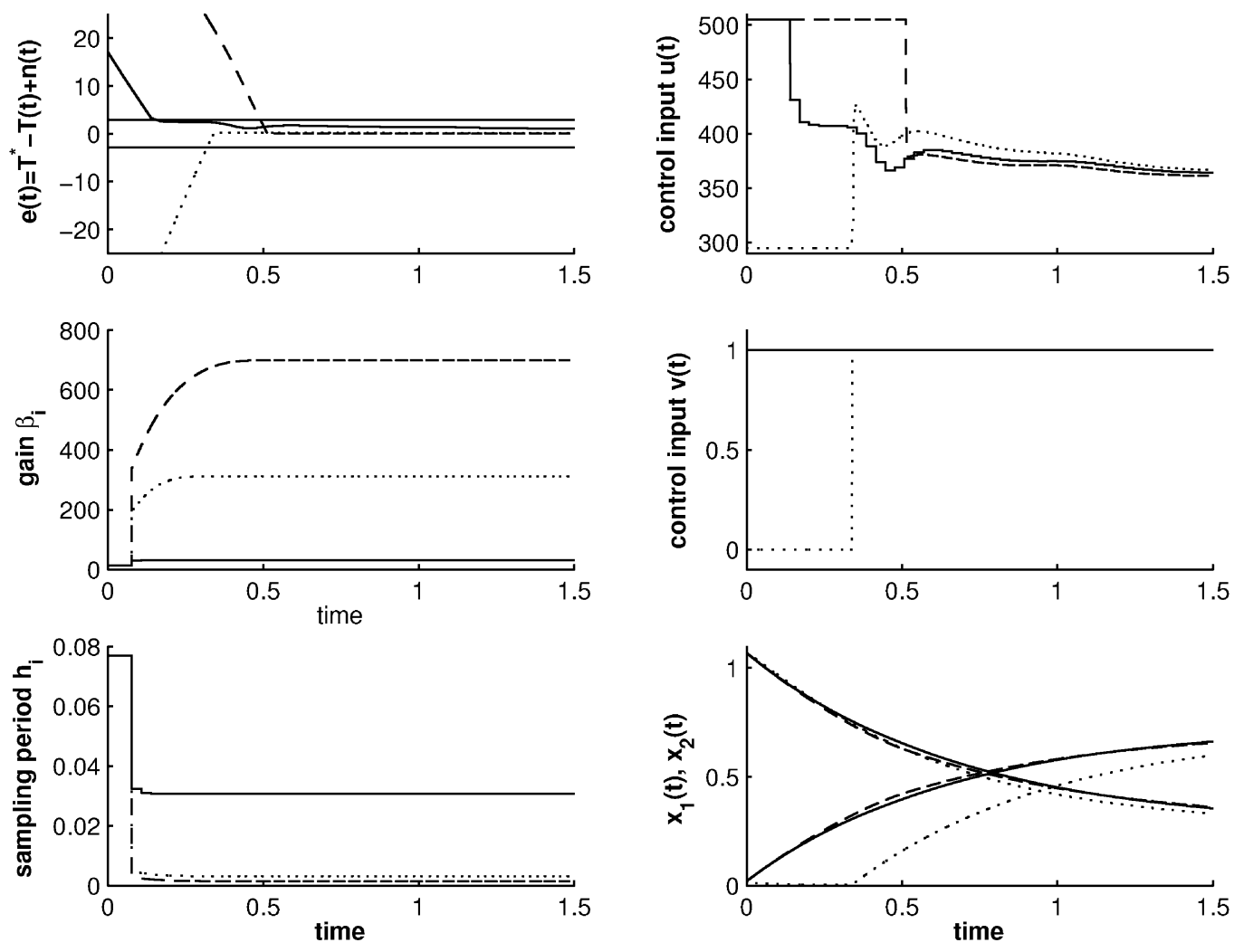

Fig. 2. Closed-loop setpoint control of the adaptive $\lambda$-tracker (7) with control parameters (16) applied to the single reaction (14) with parameters (15), in the presence of measurement noise (18), and initial temperature $T^{0}=270 \mathrm{~K}$ (dashed), $T^{0}=320 \mathrm{~K}$ (

Fig. 1 shows the simulations in the noise-free case. The final gain is only slightly larger than for continuous time adaptation and can be reduced by exploiting the flexibility of selecting $f$ and $g_{\lambda}$ in the admissible gain adaptation. Note that the gain takes on largest values for initial temperature $T^{0}=390$ which is the temperature that cannot be handled by the corresponding local adaptive controller.

Note also that in the continuous-time adaptive case, the closed-loop performance is significantly worse in the presence of noise corrupting the temperature measurement than in the noise free case, whereas in the sampled-data case the difference in performance with and without noise is not that significant, see Fig. 2. One reason for this could be that the continuous-time noise might fluctuate much more wildly than its sampled-data counterpart.

\section{Proofs}

It is worth emphasizing that there are a number of essential differences between the proofs of the sampled-data results and the analogous continuous-time result in [5, Theorem 10]. Indeed, whilst in the continuous-time case it is easy to see that the solutions exists for all time, here we have to rule out the possibility that the sampling periods have a finite-sum, i.e. $\sum_{i=0}^{\infty} h_{i}<\infty$, so that the solution only exists on a finite interval. A more awkward problem arises because of the availability of certain signals only at sampling instants. In the continuous-time proof we appeal repeatedly to the fact that certain temperature values are 'repelling' by using a Lypunov-function 
argument. In the sampled-data proof we can no longer appeal to this idea. Instead we need to work with 'repelling' intervals.

\subsection{Proof of Theorem 1}

Let $n(\cdot): \mathbb{R} \geqslant 0 \rightarrow \mathbb{R}$ denote a continuous noise satisfying (10). We may choose $\eta>0$ such that

$$
2\|n\|_{\infty}+\eta<\bar{T}-T^{*} .
$$

We proceed in several steps.

Step 1 . We show positive invariance of $\Omega\left(\gamma, x^{\text {in }}\right) \times \mathbb{R}_{>0}$ under (1). By (A1), we may consider a solution $(x, T)$ : $[0, \omega) \rightarrow \mathbb{R}_{\geqslant 0}^{n} \times \mathbb{R}_{>0}$ of (1) for some $\omega \in(0, \infty]$ and with initial data $(x(0), T(0)) \in \Omega\left(\gamma, x^{\mathrm{in}}\right) \times \mathbb{R}_{>0}$. Then integrating

$$
\frac{\mathrm{d}}{\mathrm{d} \tau} \gamma^{\mathrm{T}} x(\tau)=\gamma^{\mathrm{T}} \operatorname{Cr}(x(\tau), T(\tau))+d \gamma^{\mathrm{T}}\left[\left(\begin{array}{c}
v(\tau) \\
x_{2}^{\text {in }}
\end{array}\right)-x(\tau)\right]
$$

over $[0, t)$, for arbitrary $t \in[0, \omega)$, yields, by invoking (A2) and $\gamma^{\mathrm{T}} x(0)<\gamma^{\mathrm{T}} x^{\mathrm{in}}$,

$$
\begin{aligned}
\gamma^{\mathrm{T}} x(t) & \leqslant \mathrm{e}^{-d t} \gamma^{\mathrm{T}} x(0)+d \int_{0}^{t} \mathrm{e}^{-d(t-\tau)} \mathrm{d} \tau \gamma^{\mathrm{T}} x^{\mathrm{in}} \\
& \leqslant \gamma^{\mathrm{T}} x^{\mathrm{in}}-\mathrm{e}^{-d t}\left[\gamma^{\mathrm{T}} x^{\text {in }}-\gamma^{\mathrm{T}} x(0)\right] \leqslant \gamma^{\mathrm{T}} x^{\mathrm{in}} .
\end{aligned}
$$

This proves the claim.

Step 2 . We show that the initial value problem (1), (3)-(6), $(x(0), T(0))=\left(x^{0}, T^{0}\right)$, has a unique solution

$$
(x, T):[0, \infty) \rightarrow \Omega\left(\gamma, x^{\text {in }}\right) \times \mathbb{R}_{>0} .
$$

(2A). By (A1) and since the right-hand side of (1) is locally Lipschitz in its arguments, the theory of ordinary differential equations ensures, for any initial data $\left(x^{0}, T^{0}\right) \in \Omega\left(\gamma, x^{\text {in }}\right) \times \mathbb{R}_{>0}$, the existence of a unique solution $(x, T):\left[0, \delta_{1}\right) \rightarrow \Omega\left(\gamma, x^{\mathrm{in}}\right) \times \mathbb{R}_{>0}$ for some maximal $\delta_{1} \in\left(0, t_{1}\right]$. Since $x$ is bounded by step 1 , the affine linear boundedness of the right-hand side of (1) in $T$ ensures that the $T$-dynamics cannot produce a finite escape time. Therefore, $\delta_{1}=t_{1}$.

(2B). Consider next the initial value problem (1), $(\tilde{x}(0), \tilde{T}(0))=\left(x^{0}, T^{0}\right)$ with $u(\cdot) \equiv u_{0}, v(\cdot) \equiv v_{0}$ on $\mathbb{R} \geqslant 0$. With the same reasoning as in step $2 \mathrm{~A}$, it follows that there exists a unique solution $(\tilde{x}, \tilde{T}):[0, \infty) \rightarrow \Omega\left(\gamma, x^{\text {in }}\right) \times \mathbb{R}_{>0}$ and, in particular, $(x(t), T(t))=(\tilde{x}(t), \tilde{T}(t))$ for all $t \in\left[0, t_{1}\right)$. Therefore $\left(x_{1}, T_{1}\right):=\lim _{t \rightarrow t_{1}}(x(t), T(t))$ is well defined.

(2C). Analogous reasoning as in steps $2 \mathrm{a}$ and $2 \mathrm{~b}$ for the initial value problem (1), (3)-(6), $\left(x\left(t_{1}\right), T\left(t_{2}\right)\right)=\left(x_{1}, T_{1}\right)$ extends the solution $(x, T)$ uniquely on $\left[0, t_{2}\right)$. Proceeding inductively in this way proves that the there is a unique and maximal solution

$$
(x, T):[0, \omega) \rightarrow \Omega\left(\gamma, x^{\text {in }}\right) \times \mathbb{R}_{>0} \quad \text { for } \omega:=\sum_{i=0}^{\infty} h_{i} \in(0, \infty]
$$

of the initial value problem (1), (3)-(6), $(x(0), T(0))=\left(x^{0}, T^{0}\right)$.

2D. We show that $\omega=\infty$ in (22). Seeking a contradiction, suppose that $\omega<\infty$. Finiteness of $\omega$ in turn implies that $\sum_{i=0}^{\infty} h_{i}<\infty$ which in turn means that $\lim _{i \rightarrow \infty} h_{i}=0$. Since $\dot{T}$ is affine linearly bounded in $T$, see (1), it 
follows that $T$ is bounded on $[0, \omega)$. Therefore, $\left(T_{i}\right)$ is bounded and thus there exists $\hat{M}>0$ so that $g_{\lambda}\left(e_{i}\right) \leqslant \hat{M}$ for all $i \in \mathbb{N}$. Using (5), this implies that

$$
\beta_{i+1} \leqslant \beta_{0}+\hat{M} \sum_{j=0}^{i} h_{j} \quad \text { for all } i \in \mathbb{N}
$$

which yields boundedness of $\left(\beta_{i}\right)$, and thus $\left(h_{i}\right)=\left(f\left(\beta_{i}\right)\right)$ is uniformly bounded away from 0 , which contradicts the assumption. This proves step $2 \mathrm{D}$ and completes the proof of step 2.

From now on fix $M$ as in (12).

Step 3. We show that boundedness of $\left(\beta_{i}\right)$ yields Assertions (i)-(iv). If $\left(\beta_{i}\right)$ is bounded, then by monotonicity we have (ii); and furthermore $\left(h_{i}\right)=\left(f\left(\beta_{i}\right)\right)$ is uniformly bounded away from 0 , whence (iii). Now (5) ensures that

$$
h_{\infty} \sum_{i=0}^{\infty} g_{\lambda}\left(e_{i}\right) \leqslant \sum_{i=0}^{\infty} h_{i} g_{\lambda}\left(e_{i}\right)=\beta_{\infty}-\beta_{0} \in \mathbb{R} .
$$

Therefore $\lim _{i \rightarrow \infty} g_{\lambda}\left(e_{i}\right)=0$, and (6a), (6b) give $\lim _{i \rightarrow \infty} \operatorname{dist}\left(\left|e_{i}\right|,[0, \lambda]\right)=0$, which proves (iv). We then have that $\left(T_{i}\right)$ is bounded, and therefore boundedness of $T(\cdot)$ on $[0, \infty)$ is a consequence of (13), whence (i).

Step 4 . If $\left(\beta_{i}\right)$ is unbounded, then we may choose $i_{0} \in \mathbb{N}$ so that

$$
\beta_{i} \geqslant \max \left\{\frac{2\left(u^{*}-\underline{u}\right)}{\lambda-2\|n\|_{\infty}}, \frac{2\left(\bar{u}-u^{*}\right)}{\lambda-2\|n\|_{\infty}}, \frac{u^{*}-\underline{u}}{\eta}\right\}, \quad \forall i \geqslant i_{0}
$$

holds. Note that if (23) does not hold for any $i_{0} \in \mathbb{N}$, then, by invoking that $\left(\beta_{i}\right)$ is a non-decreasing sequence, it follows that $\left(\beta_{i}\right)$ is bounded, and (i)-(iv) are a consequence of Step 3.

Step 5. We show that if $\left(\beta_{i}\right)$ is unbounded, then there exists $i_{1} \geqslant i_{0}$ such that $T_{i_{1}} \in(0, \bar{T}]$. Suppose $\left(\beta_{i}\right)$ is unbounded and let $i_{0}$ be such that (23) holds.

(5A). We show

$$
\left[i \geqslant i_{0} \wedge T_{i}>\bar{T}\right] \Longrightarrow\left[\forall t \in\left[t_{i}, t_{i+1}\right): u(t)=\underline{u} \wedge v(t)=0\right] .
$$

Indeed, if $T_{i}>\bar{T}$ and $i \geqslant i_{0}$, then

$$
\beta_{i} \mathrm{e}_{i}+u^{*} \stackrel{(2)}{\leqslant} \beta_{i}\left[T^{*}-\bar{T}+\|n\|_{\infty}\right]+u^{*} \stackrel{(20)}{\leqslant}-\beta_{i} \eta+u^{*} \stackrel{(23)}{\leqslant} \underline{u}
$$

which, applied to (6), yields (24).

(5B). We show that

$$
\left[\forall t \in\left[t_{i}, t_{i+1}\right): v(t)=0\right] \Longrightarrow\left[\forall t \in\left[t_{i}, t_{i+1}\right):\left\|x_{1}(t)\right\| \leqslant \mathrm{e}^{-d\left(t-t_{i}\right)}\left\|x_{1}\left(t_{i}\right)\right\|\right] .
$$

Since all entries of $C^{1}$ are non-positive, (1) gives, for all $\tau \in\left[t_{i}, t_{i+1}\right)$,

$$
\frac{\mathrm{d}}{\mathrm{d} \tau}\left\|x_{1}(\tau)\right\|^{2}=2 x_{1}(\tau)^{\mathrm{T}}\left[C^{1} r(x(\tau), T(\tau))-d x_{1}(\tau)\right] \leqslant-2 d\left\|x_{1}(\tau)\right\|^{2},
$$

and the claim follows by integration over $\left[t_{i}, t\right)$.

(5C). Finally, seeking a contradiction to the claim of step 5, assume that $T_{i}>\bar{T}$ for all $i \geqslant i_{0}$.

By (A3), we may choose $\varepsilon \in(0, q)$ sufficiently small so that

$$
-[q-\varepsilon] \bar{T}+\underline{u}<-\rho .
$$

Note that (24) together with (25) yields

$$
\left\|x_{1}(t)\right\| \leqslant \mathrm{e}^{-d\left(t-t_{i_{0}}\right)}\left\|x_{1}\left(t_{i_{0}}\right)\right\| \quad \text { for all } t \in\left[t_{i_{0}}, \infty\right),
$$


and hence, by (A4), there exists $t^{*} \geqslant t_{i_{0}}$ so that

$$
\hat{r}\left(x_{1}(t)\right) \leqslant \varepsilon /\|b\| \text { for all } t \geqslant t^{*} .
$$

Therefore,

$$
\dot{T}(t) \stackrel{(24)}{=} b^{\mathrm{T}} r(x(t), T(t))-q T(t)+\underline{u} \leqslant\|b\| \hat{r}\left(x_{1}(t)\right) T(t)-q T(t)+\underline{u} \stackrel{(28)}{\leqslant}-[q-\varepsilon] T(t)+\underline{u},
$$

and hence it follows for $t \geqslant t^{*}$ that whilst $T(t)>\bar{T}$, we have

$$
\dot{T}(t) \leqslant-[q-\varepsilon] \bar{T}+\underline{u} \stackrel{(27)}{<}-\rho .
$$

This contradicts the assumption and completes the proof of step 5.

Step 6. Suppose $\left(\beta_{i}\right)$ is unbounded. We show that

$$
\exists i_{1} \geqslant i_{0}:\left[T_{i_{1}} \in(0, \bar{T}] \Longrightarrow\left[\forall t \in\left[t_{i_{1}}, \infty\right): T(t) \in(0, \bar{T}]\right]\right] .
$$

(6A). Let $i \geqslant i_{0}$ and $T_{i} \in\left[\bar{T}-\|n\|_{\infty}, \bar{T}\right]$. We show that if $t \in\left[t_{i}, t_{i+1}\right)$ and $T(t) \in\left[\bar{T}-\|n\|_{\infty}, \bar{T}\right]$, then $\dot{T}(t)<-\rho$. Indeed, since $\bar{T}-\|n\|_{\infty} \leqslant T_{i}$ we have

$$
\beta_{i} \mathrm{e}_{i}+u^{*} \stackrel{(2)}{\leqslant} \beta_{i}\left[T^{*}-T_{i}+\|n\|_{\infty}\right]+u^{*} \leqslant \beta_{i}\left[T^{*}-\bar{T}+2\|n\|_{\infty}\right]+u^{*} \stackrel{(20)}{\leqslant}-\beta_{i} \eta+u^{*} \stackrel{(23)}{\leqslant} \underline{u},
$$

so that

$$
\dot{T}(t)=b^{\mathrm{T}} r(x(t), T(t))-q T(t)+\underline{u} \text { for all } t \in\left[t_{i}, t_{i+1}\right) .
$$

If $\bar{T}-\|n\|_{\infty} \leqslant T(t)$, then (10) yields $T^{*}+2\|n\|_{\infty}<\bar{T} \leqslant T(t)+\|n\|_{\infty}$, and so $\underline{T} \leqslant T^{*}+\|n\|_{\infty}<T(t) \leqslant \bar{T}$, and we may apply (A3) to (30) to conclude that $\dot{T}(t)<-\rho$.

(6B). Finally, to show step 6 , assume that there exists $i \geqslant i_{0}$ such that $T_{i} \in(0, \bar{T}]$ and consider two cases:

(i) If $T_{i} \in\left[\bar{T}-\|n\|_{\infty}, \bar{T}\right]$, then step 6A ensures that $T(t)$ moves to the left if $t \in\left[t_{i}, t_{i+1}\right)$ and $T(t) \in\left[\bar{T}-\|n\|_{\infty}, \bar{T}\right]$. Therefore, $T(t) \leqslant \bar{T}$ over the period $\left[t_{i}, t_{i+1}\right)$.

(ii) Suppose that $T_{i} \in\left(0, \bar{T}-\|n\|_{\infty}\right]$. By (13), one may choose $h>0$ such that

$$
\left[\exists t^{*} \geqslant 0: T\left(t^{*}\right) \in\left(0, \bar{T}-\|n\|_{\infty}\right]\right] \Longrightarrow\left[\forall t \in\left[t^{*}, t^{*}+h\right):\left|T(t)-T\left(t^{*}\right)\right|<\|n\|_{\infty}\right] .
$$

Now one may choose $i_{1} \in \mathbb{N}$ such that $h_{i}<h$ for all $i \geqslant i_{1}$. It then follows that

$$
\left[\forall i \geqslant i_{1}: T_{i} \in\left(0, \bar{T}-\|n\|_{\infty}\right]\right] \Longrightarrow\left[T_{i+1}<\bar{T}\right] .
$$

This completes the proof of step 6 .

Step 7. To show boundedness of $\left(\beta_{i}\right)$, we prove that unboundedness of $\left(\beta_{i}\right)$ yields

$$
\exists i_{3} \geqslant i_{1} \quad \forall i \geqslant i_{3}: \quad \beta_{i}=\beta_{i_{3}}
$$

whence $\left(\beta_{i}\right)$ is bounded.

Suppose $\left(\beta_{i}\right)$ is unbounded. Then steps 4,5 , and 6 guarantee that

$$
\forall t \in\left[t_{i_{1}}, \infty\right): T(t) \in(0, \bar{T}] .
$$

By (13) there exists $h>0$ such that

$$
\forall h_{i}<h:\left|T_{i+1}-T_{i}\right|<\lambda / 2 .
$$

If $\lim _{i \rightarrow \infty} h_{i} \geqslant h$, then $\left(\beta_{i}\right)$ is bounded, and (i)-(iv) are a consequence of step 3. Hence we may suppose that there exists $i_{2} \geqslant i_{1}$ such that $h_{i_{2}}<h$, which in turn means that $h_{i}<h$, for all $i \geqslant i_{2}$. 
(7A). We show that

$$
\left[\exists i \geqslant i_{2}: T_{i}<T^{*}-\frac{\lambda}{2}\right] \Longrightarrow\left[\forall t \in\left[t_{i}, t_{i+1}\right): \dot{T}(t)>\rho\right]
$$

and

$$
\left[\exists i \geqslant i_{2}: T^{*}+\frac{\lambda}{2}<T_{i}\right] \Longrightarrow\left[\forall t \in\left[t_{i}, t_{i+1}\right): \dot{T}(t)<-\rho\right] .
$$

Let $T_{i}<T^{*}-\lambda / 2$ and $i \geqslant i_{2}$. Then, by (23), we have $\beta_{i} \mathrm{e}_{i}+u^{*} \geqslant \bar{u}$, and so $u(t)=\bar{u}$ for all $t \in\left[t_{i}, t_{i+1}\right)$, which gives, by invoking (32) and (A3),

$$
\dot{T}(t)=b^{\mathrm{T}} r(x(t), T(t))-q T(t)+\bar{u} \geqslant \rho \quad \forall t \in\left[t_{i}, t_{i+1}\right) .
$$

The second implication follows similarly.

(7B). Finally, if $i \geqslant i_{2}$ and $T_{i} \notin\left[T^{*}-\lambda / 2, T^{*}+\lambda / 2\right]$, then by step 7A together with (33) it follows that there exists $i_{3} \geqslant i_{2}$ such that $T_{i_{3}} \in\left(T^{*}-\lambda / 2, T^{*}+\lambda / 2\right)$. Invoking (33) again yields $T_{i_{3}+1} \in\left(T^{*}-\lambda, T^{*}+\lambda\right)$, and therefore we have, for all $i \geqslant i_{3}$, that $T_{i} \in\left(T^{*}-\lambda, T^{*}+\lambda\right)$, whence (31). This completes the proof of Step 7 and also the proof of the theorem.

\subsection{Proof of Theorem 2 and Propositions 3 and 4}

These proofs are both a straightforward modification of the proof of Theorem 1, essentially replacing sequences $\left(\beta_{i}\right)$ and $\left(h_{i}\right)$ by sufficiently large respectively small constants.

\section{Acknowledgements}

We are indebted to Thomas Müller (Ilmenau) for carefully performing the simulations.

\section{References}

[1] G. Bastin, D. Dochain, On-Line Estimation and Adaptive Control of Bioreactors, Elsevier Science Publishers B.V., Amsterdam, 1990.

[2] C.I. Byrnes, J.C. Willems, Adaptive stabilization of multivariable linear systems, Proceedings of the 23rd Conference on Decision and Control, Las Vegas, 1984, 1574-1577.

[3] G.R. Gavalas, Nonlinear Differential Equations of Chemically Reacting Systems, Springer, Berlin, 1968.

[4] A. Ilchmann, E.P. Ryan, Universal $\lambda$-tracking for nonlinearly perturbed systems in the presence of noise, Automatica 30 (1994) $337-346$.

[5] A. Ilchmann, M. Thuto, S. Townley, Input constrained adaptive tracking with applications to exothermic chemical models, SIAM J. Control Optim. 43 (2004) 154-173.

[6] A. Ilchmann, S. Townley, Adaptive high-gain $\lambda$-tracking with variable sampling rate, Systems Control Lett. 36 (1999) $285-293$.

[7] A. Ilchmann, S. Trenn, Input constrained control with applications to chemical reactor models, Systems Control Lett. 53 (2004) $327-424$.

[8] A. Ilchmann, M.-F. Weirig, Modelling of general biotechnological processes, Math. Comput. Modelling Dynam. Syst. 5 (1999) $152-178$.

[9] F. Jadot, G. Bastin, F. Viel, Robust global stabilization of stirred tank reactors by saturated output feedback, European J. Control 5 (1999) 361-371.

[10] I. Mareels, A simple selftuning controller for stably invertible systems, Systems Control Lett. 4 (1984) 5-16.

[11] D.E. Miller, E.J. Davison, An adaptive controller which provides an arbitrarily good transient and steady-state response, IEEE Trans. Automat. Control 36 (1991) 68-81.

[12] A.S. Morse, Recent problems in parameter adaptive control, in: I.D. Landau (Ed.), Outils et Modèles Mathématiques pour l'Automatique l'Analyse de Systèmes et le Traitment du Signal, Editions du CNRS 3, Paris, 1983, pp. 733-740.

[13] C. Sparrow, The Lorenz Equations: Bifurcations, Chaos, and Strange Attractors, Springer, New York, 1982.

[14] F. Viel, F. Jadot, G. Bastin, Global stabilization of exothermic chemical reactors under input constraints, Automatica 33 (1997) $1437-1448$. 